\title{
Plethysmographic validation of near infrared spectroscopic monitoring of cerebral blood volume
}

\author{
Y A B D Wickramasinghe, L N Livera, S A Spencer, P Rolfe, M S Thorniley
}

\begin{abstract}
The validation of measurement of cerebral blood volume (CBV) and cerebral blood flow (CBF) using near infrared spectroscopy (NIRS) against jugular venous occlusion plethysmography is described. Repeated measurements in six infants were made using both techniques simultaneously. A close relationship between the two measurements of change in CBV was obtained in five infants. There was also a close relationship for measurement of CBF in four infants. This study confirms the possibility of using NIRS to monitor CBV continuously in the premature infant. This parameter may prove to be of greater clinical value than the intermittent measurement of CBF.
\end{abstract}

Near infrared spectroscopy (NIRS) is a new technique originally proposed by Jobsis in 1977 for continuous monitoring of cerebral haemoglobin concentration and oxygenation. ${ }^{1}$ The NIRS instrumentation developed in this department has been used to study these cerebral parameters continuously by transillumination of the neonatal head. Algorithms are used to convert the changes in absorbance at individual wavelengths to changes in the concentration of oxygenated, deoxygenated, and total haemoglobin. ${ }^{2}$ Assuming a constant packed cell volume, a changed in total haemoglobin implies a change in cerebral blood volume (CBV). Clinical studies using this technique have demonstrated that, whereas mild hypoxaemia leads to an increase in CBV, bradycardia causes a rapid fall with a small overshoot on recovery. ${ }^{3}$ Changes in CBV attributable to alterations in carbon dioxide tension have also been documented. ${ }^{4}$ Although the magnitude of these changes can be calculated from theoretical considerations, the technique for measurement of CBV has never been validated by an independent technique.

Strain gauge plethysmography has been used to calculate cerebral blood flow (CBF) from a consideration of change in cerebral volume in relation to time. ${ }^{6}$ Although the technique has been criticised for this purpose, it is generally accepted that an accurate measurement of increase in head circumference resulting from jugular venous occlusion does provide a useful method of quantifying volume change.

\section{Methods \\ INSTRUMENTATION \\ NIRS}

This technique uses near infrared light emitted by three semiconductor laser diodes to transilluminate the neonatal head. ${ }^{7-9}$ The near infrared light from the semiconductor diodes is coupled to an optical fibre, which is attached to the head. The light emerging after absorption is collected by a similar optical fibre bundle, detected by an avalanche photodiode and amplified. After initial calibration, the overall absorbance at each of the three wavelengths $(775 \mathrm{~nm}$, $845 \mathrm{~nm}$, and $904 \mathrm{~nm}$ ) can be determined. The principle behind the technique is that the absorbance at these wavelengths is sensitive to the concentrations of oxygenated and deoxygenated haemoglobin in the brain.

For the analysis a modified form of LambertBeer law is assumed to hold for biological tissue. In its simplest form this may be expressed as:

$$
\mathrm{A}=\mathrm{eCnL}+\mathrm{B}
$$

where, A is the optical absorption (optical density units) of the chromophore of concentration $\mathrm{C}(\mathrm{mmol} / \mathrm{l})$ having an absorption coefficient $\mathrm{e}$ $(\mathrm{l} / \mathrm{cm} \times \mathrm{mmol})$ and a physical path length $\mathrm{L}$ (cm); and B is a term to account for the losses in absorption due to the skin-probe interface and scattering. The optical path length is expressed as $\mathrm{nL}$ where $\mathrm{n}$ is a path length multiplication factor used to take into account the increase in optical pathlength due to scattering, and we have used a factor of $4 \cdot 3$. $^{4}$

Thus change in absorbance can be related to change in concentration as:

$$
\Delta \mathrm{A}=\Delta \mathrm{C}(\mathrm{enL})
$$

provided the assumption is made that scattering and losses at the skin-fibre interface remains unchanged during the study period.

Thus if $\Delta \mathrm{A}_{775}, \Delta \mathrm{A}_{845}$, and $\Delta \mathrm{A}_{904}$ are the changes in absorbances at these wavelengths then change in total haemoglobin (HbT) is given by:

$$
\begin{gathered}
\Delta[\mathrm{HbT}]=\left[(0.485)\left(\Delta \mathrm{A}_{775}\right)+(0.861)\left(\Delta \mathrm{A}_{845}\right)+\right. \\
\left.(1.249)\left(\Delta \mathrm{A}_{904}\right)\right] / \mathrm{nL} \mathrm{mmol} / \mathrm{l}
\end{gathered}
$$

where $\mathrm{L}$ is the direct spacing between the optical probes.

NIRS cannot be used continuously to monitor absolute CBV, but can be used to monitor changes in the total haemoglobin in the neonatal brain. The change in total haemoglobin is normally given as $\mathrm{mmol} / \mathrm{l}$ but can be converted to blood volume if the packed cell volume is known, and assuming a cerebral to large vessel packed cell volume ratio of $0 \cdot 7 .{ }^{4}$ The molecular weight of haemoglobin is taken to be 64500 . Thus the change in CBV may be expressed as 
$\mathrm{ml} / 100 \mathrm{ml}$. The CBF can be calculated from the rate of change of blood volume as monitored by NIRS, and expressed as $\mathrm{ml} / 100 \mathrm{ml} / \mathrm{min}$.

\section{Strain gauge plethysmography}

Strain gauge plethysmography employs a Wheatstone bridge to determine the changes in length of the gauge. ${ }^{10} 11$ This technique can be adapted to determine CBF. ${ }^{1213}$ For neonatal cerebral studies the strain gauge consists of a silicone rubber tube of internal diameter $1 \mathrm{~mm}$ and length $50 \mathrm{~cm}$ which is filled with mercury. The tube is doubled on itself to form a four stranded gauge. Copper pins are attached to the ends for electrical connections, and connected to one arm of the bridge circuit.

The electronic circuit produces a voltage change which is proportional to the change in occipitofrontal head circumference of the baby, when the jugular veins are occluded. The near infrared data and strain gauge information can be displayed and stored simultaneously.

The technique relies on the compliance of the neonatal skull due to the softness of the bones and lack of fusion of the sutures. It is assumed that any change in CBV causes the entire head to expand or contract isotropically. The change in head circumference when the overall length of the gauge is changed by $1 \mathrm{~mm}$ can be determined in situ with the calibration device. Provided the head circumference is known, the change in CBV as a result of jugular venous occlusion can be calculated as given below. The rate of change of CBV can also be calculated to give a measure of apparent CBF. This acknowledges the assumptions inherent in the technique, which tend to lead to an underestimate of flow. ${ }^{6}$

Cooke $e t$ al have reported that the relationship between brain weight and occipitofrontal head circumference is cubic in nature ${ }^{12}$ :

$$
\text { Brain weight }=C^{3.001} \times 0.009
$$

where $\mathrm{C}$ is the occipitofrontal circumference. As the relationship between brain weight and volume $(\mathrm{V})$ is linear, and also assuming that the density of brain is 1.06 , the brain volume is given by:

$$
\mathrm{V}=\mathrm{C}^{3} \times 0.009 / 1 \cdot 06=0.0085 \times \mathrm{C}^{3}
$$

For a small change in blood volume:

$$
d V=3 \times 0.0085 \times C^{2} d C=0.0255 \times C^{2} d C
$$

Apparent CBF is determined as the rate of change of blood volume, and is given by:

$$
\mathrm{dV} / \mathrm{dt}=(3 \times \mathrm{V} \times 60 / \mathrm{C}) \times \mathrm{dC} / \mathrm{dt} \mathrm{ml} / \mathrm{min}
$$

For $100 \mathrm{ml}$ of brain tissue:

$$
\text { Apparent } \mathrm{CBF}=\left(\begin{array}{c}
3 \times 100 \times 60 / \mathrm{C}) \\
\mathrm{ml} / \mathrm{min} .
\end{array}\right.
$$

Hence the slope of the blood volume change $(\mathrm{dC} / \mathrm{dt})$ provides a means of determining the apparent CBF.

\section{PATIENTS AND PROCEDURES}

Six premature infants were studied. These were all infants who had had no major neonatal problems and had normal cranial ultrasound scans at the time of the study. Gestation ranged from 31 to 33 weeks (median 32 weeks) and birth weight from 1540 to $2010 \mathrm{~g}$ (median $1735 \mathrm{~g}$ ). Age at the time of study was from 3 to 16 days, median 15 days.

The infants were studied after a feed, while at rest or in quiet sleep. The head circumference was measured, the strain gauge was then placed around the occipitofrontal circumference and fastened using Velcro tape. The NIRS probes were held in place a measured distance apart using double sided adhesive rings and elastic webbing. Great care was needed to ensure that that strapping for the NIRS probes did not touch or interfere with the strain gauge. The baby was then positioned on the lap of one investigator with the head supported by a roll of cloth. The connecting wires and the NIRS optical fibres were supported to prevent any tension.

With the equipment in position and the infant at rest, small pulsations were obtained on the strain gauge trace, due to the cardiac cycle (fig 1). These were not seen on the NIRS traces because of the two second averaging time used. Occlusions were performed as follows: the two jugular veins in the lower third of the neck were occluded simultaneously using the index fingers for a period of five seconds, then released. Once the trace had returned to baseline, the occlusion was repeated, to approximately 10 occlusions. A calibration manoeuvre was then performed. In some infants it was not possible to analyse 10 occlusions because of movement artefact, and the number ranged from five to seven occlusions per patient.

This study was approved by the local ethical committee and informed consent was obtained from parents.

\section{Results}

A typical study is shown in fig 2. This was

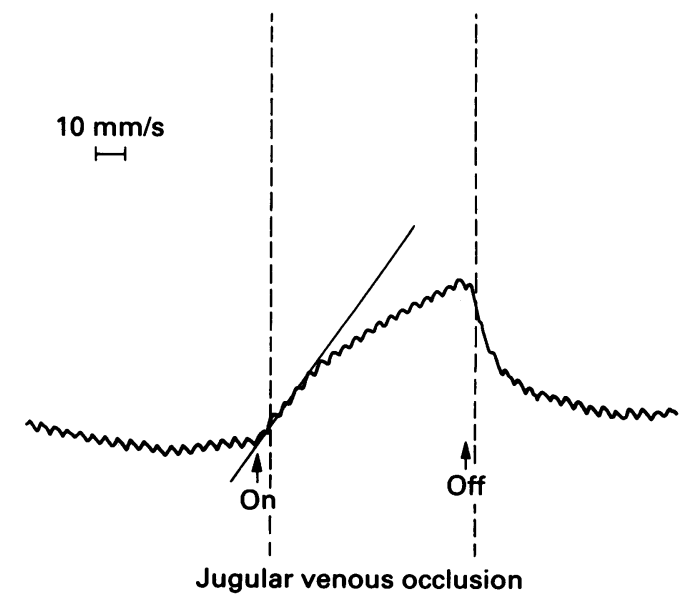

Figure 1 This shows the direct trace from strain gauge plethysmography in an infant of 32 weeks' gestation at rest. Individual cardiac pulsations can clearly be seen together with the increase in occipitofrontal circumference with jugular with the increase in occipitofrontal circumference wion is
venous occlusion and the decrease when the occlusion is released. The oblique line indicates the slope used for calculation of apparent $C B F$. 
carried out on an infant of 32 weeks' gestation, birth weight $1670 \mathrm{~g}$, at the age of 15 days. Several jugular venous occlusions were carried out to determine the CBV and flow. The two traces showing the variations of $\mathrm{CBV}$ as measured by NIRS and by strain gauge can be seen to correspond.

Figure 3 shows a scatter diagram, with the line of identity, of CBV data plotted for each of the six babies. The data for five babies shows a relationship between the two methods for the magnitude of change in CBV observed during an occlusion. Results from five occlusions in one baby (baby 3 ) did not fit with this relationship. The mean change in CBV during a single jugular venous occlusion as measured by NIRS, excluding the data from baby 3 , was $1 \cdot 13$ $\mathrm{ml} / 100 \mathrm{ml}$, while that measured by the strain gauge techique was $1.25 \mathrm{ml} / 100 \mathrm{ml}$.

Friedman two way analysis of variance by ranks as modified by Conover ${ }^{14}$ was carried out

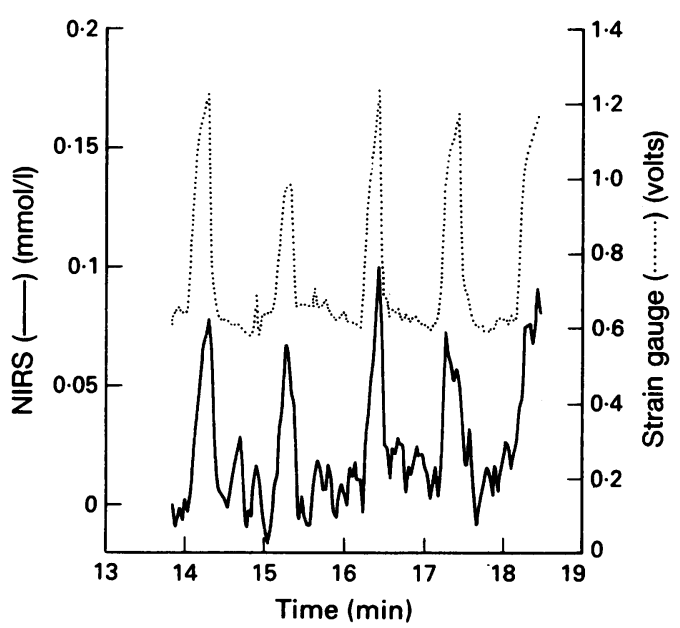

Figure 2 This demonstrates simultaneous recording of occipitofrontal circumference by strain gauge and $C B V$ by NIRS during several jugular venous occlusions.

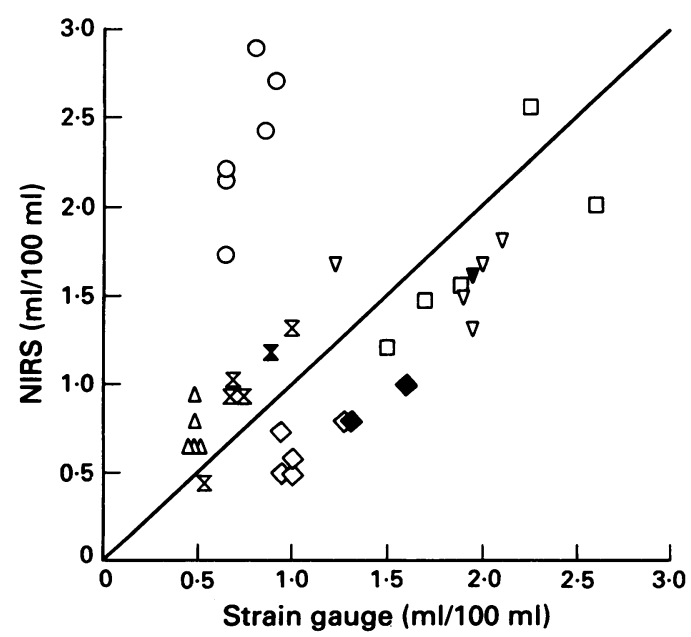

$$
\begin{array}{lll}
\Delta \text { Baby } 1 & \text { \& Baby } 2 & \circ \text { Baby } 3 \\
\square \text { Baby } 4 & \nabla \text { Baby } 5 & \diamond \text { Baby } 6
\end{array}
$$

Figure 3 NIRS measurement of changes in $C B V$ is shown plotted against strain gauge measurement for six babies individually identified. The solid symbols are data points not used in statistical analysis. on the data for several observations per experimental unit. The data from baby 3 was excluded from analysis (see discussion). This analysis demands equal number of observations from each individual and therefore the first five successful occlusions from each set were used. Here the test statistic calculated is compared with $\chi^{2}$ for degrees of freedom (df), $k-1$, where $k$ is the number of treatment (measurement) categories (quoted figures: $\chi_{\mathrm{df}=1}^{2}=3.84$ for $p=0.05$ and 1.32 for $p=0.25$ ). The calculated test statistics for change in CBV was 0.375 , which is not significant. The test demonstrates that null hypothesis appears to be valid $(p>>0.25)$ and any deviation from agreement between the methods is not significant.

CBF was calculated from each occlusion using the two methods described above. The results from four babies are shown in fig 4. In the two remaining babies, CBF could not be calculated because of an artefact in the tracing. In this study low values for apparent CBF were obtained, by both methods when compared with previous published data. ${ }^{15}$ The mean apparent $\mathrm{CBF}$ measured by strain gauge was $9.73 \mathrm{ml} / 100 \mathrm{ml} / \mathrm{min}$, while the mean $\mathrm{CBF}$ calculated from the NIR data was $9.05 \mathrm{ml} / 100$ $\mathrm{ml} / \mathrm{min}$.

Using the same statistical analysis as the CBV the calculated test statistics for CBF was $0 \cdot 01$, which again demonstrates that any deviation from agreement between the two methods is not significant.

\section{Discussion}

Disturbance to cerebral blood flow is likely to be an important factor in the aetiology of neonatal cerebral injury. Therefore a number of different methods have been developed for the assessment of CBF in premature infants.

The problems inherent in the technique of jugular venous occlusion plethysmography for the measurement of $\mathrm{CBF}$ have been described. ${ }^{13}$

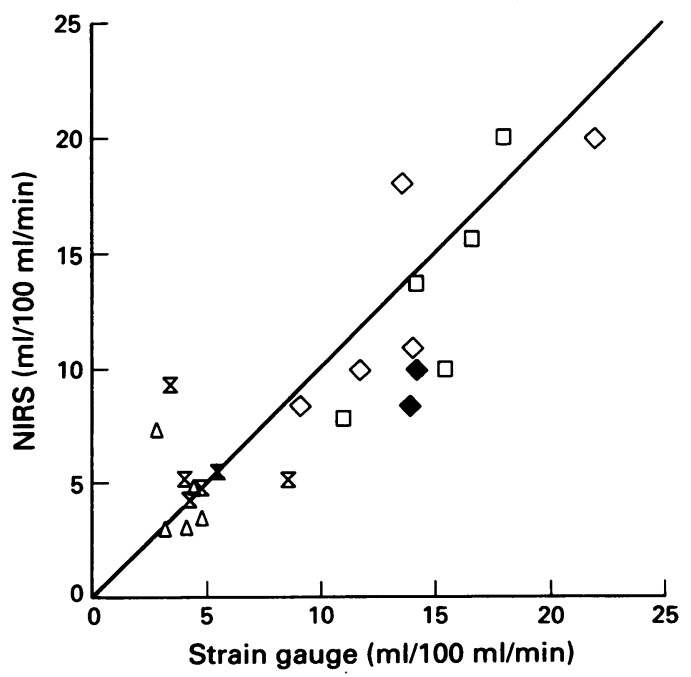

$\triangle$ Baby $1 \quad \&$ Baby $2 \quad \square$ Baby $4 \diamond$ Baby 6

Figure 4 Data for $C B F$ measurements by the two methods is shown for four babies (in the remaining two CBF could not be calculated). The solid symbols are data points not used in statistical analysis. 
The validity of the estimation of CBF depends upon assumptions that cerebrospinal fluid is not displaced and only a small volume of blood escapes through the vertebral veins during an occlusion. The skull is assumed to be perfectly compliant. Other limitations include the practical difficulties of positioning the infant correctly and effecting a good clean occlusion. A rise in venous pressure is a necessary consequence of the technique and although this is well tolerated by healthy infants, it may be hazardous in sick infants who may already be hypoxic or hypotensive or have raised intracranial pressure. Concern has also been expressed over the use of mercury which certainly should not be used in an enclosed space such as an incubator. In summary, an estimate of global blood flow can be obtained within a few seconds, although the average of results from several jugular occlusions is required to obtain adequate precision. However, the coefficient of variation of flow by this technique has been quoted as $15 \%$ to $35 \%$. $^{16}$

With the exception of possible problems with skull compliance, these limitations do not apply to the use of the technique to measure changes in cerebral blood volume in healthy preterm infants. Therefore it was felt that this techique could effectively be used to validate the estimation of changes in cerebral blood volume observed using NIRS

Examination of the raw data (fig 2) clearly demonstrates a synchronous change in occipitofrontal circumference and total haemoglobin in response to each venous occlusion. There was a close relationship between the results from the techniques for the magnitude of change observed in five infants. The infant with outlying results was larger than the others (birth weight $2010 \mathrm{~g}$ ) and may therefore have had a less compliant skull leading to an underestimated change from the strain gauge measurement.

The change in path length which occurs during jugular venous occlusion also needs to be considered for its effect on NIRS measurements. For ease of calculation, assumption is made that the neonatal head could be approximated to a sphere with a circumference of $30 \mathrm{~cm}$ (fig 5).

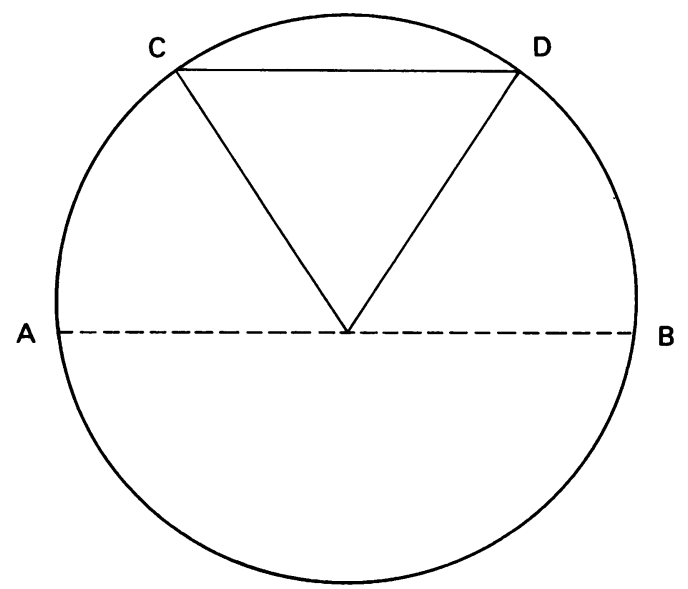

Figure 5 Change in optical path length due to jugular venous occlusion (head approximated to a sphere). $A B=$ diameter; $C$ and $D$ indicate position of NIRS probes, arc length $5 \mathrm{~cm}$; occipitofrontal circumference $=30 \mathrm{~cm}$. Angle subtended by $C D$ at the centre is $60^{\circ}$.
The change in occipitofrontal circumference after jugular venous occlusion lasting about six seconds is taken to be $1 \mathrm{~mm}$ in this exampleassuming the skull to be perfectly compliant. The change in diameter for this circumferential change is from 95.45 to $95.77 \mathrm{~mm}$. The optical probes for studies on neonates undergoing jugular venous occlusion are taken to be about $5 \mathrm{~cm}$ apart for this calculation. If it is assumed that the angle subtended is $60^{\circ}$, the change in physical path length is from $47 \cdot 72$ to $47 \cdot 88 \mathrm{~mm}$. This artifactual change is additive and will be reflected in the calculated haemoglobin concentration and hence will result in an overestimation of change in CBV up to $25 \%$ on babies with fully compliant skull. This error will be less on babies that have a less compliant skull.

Measurement of cerebral blood flow using NIRS gave comparable results to the strain gauge measurements، However, the limitations of using jugular venous occlusion remain. All methods of measuring $\mathrm{CBF}$ involve some form of intervention. A technique described by Wyatt et al using a small change in arterial oxygen saturation in conjunction with NIRS certainly causes less disturbance to the infant, ${ }^{4}$ but the fact that a change is necessary means that CBF cannot be continuously monitored.

We have shown that CBV can be monitored non-invasively and continuously using NIRS. Studies using this technique have shown that measure of $\mathrm{CBV}$ is sensitive to changes that are known to cause changes in flow. These include bradycardia, hypoxaemia, and changes in carbon dioxide tension.

If our understanding of the aetiology of cerebral ischaemic lesions occurring during intensive care is to be increased then a continuous measurement is essential. A continuous measurement of CBV may prove to be of greater value than intermittent measurements of flow.

We would like to thank the parents who gave their consent to our studies, and acknowledge the statistical consultations with Dr J Thompson and assistance of Ralph Houston and Farouk Faris in this study. The technical support given by $R$ Bailey and $T$ Buttery in designing the strain gauge calibration device is also acknowledged.

This work was supported by the Science and Engineering This work was supported by the Science and Engineering
Research Council, North Staffordshire Health Authority, and the Research Council, North Staffordshire
North Staffordshire Medical Institute.

1 Jobsis FF. Non-invasive infra red monitoring of cerebral and myocardial oxygen sufficiency and circulatory parameters. myocardial oxygen sufficien
Science 1977;198:1264-6.

2 Wickramasinghe YABD, Thorniley MS, Rolfe P, Livera LN Spencer SA. Development of algorithms for in-vivo noninvasive monitoring in biological tissue using near infra red spectroscopy. Proceedings of the Fifth Mediterranean Conspectroscopy. Proceedings of the Fifth Mediterranean Con-
ference on Medical and Biological Engineering. Greece, ference on

3 Livera LN, Spencer SA, Thorniley MS, Wickramasinghe YABD, Rolfe P. The effects of hypoxaemia and bradycardia on neonatal cerebral haemodynamics. Arch Dis Child 1991;66:376-80.

4 Wyatt JS, Edwards AD, Azzopardi D, Reynolds EOR. Magnetic resonance and near infra red spectroscopy for investigation of perinatal hypoxic-ischaemic brain injury. Arch Dis Child 1989;64:953-63.

5 Wyatt JS, Cope M, Deply DT, Wray S, Reynolds EOR. Quantification of cerebral oxygenation and haemodynamics in sick newborn infants by near infra red spectrophotometry. Lancet 1986;ii:1063-6.

6 Cooke RWI, Costeloe K, Rolfe P, Tizard JPM. Measurement of cerebral blood flow in the newborn. In: Stern L, Oh W, Friis-Hansen B, eds. Intensive care in the newborm. 2nd Ed. New York: Masson Publishing, 1978:127-33.

7 Rea PA, Crowe J, Wickramasinghe YABD, Rolfe P. Noninvasive optical methods for the study of cerebral metabolism in the human newborn: a technique for the future? $7 \mathrm{Med}$ Eng Technol 1985;9,4:160-6. 
8 Wickramasinghe YABD, Crowe J, Rolfe P. Optical method adaptable for cerebral monitoring in the newborn. Med Biol Eng Comput 1985;23,1:468-9.

9 Thorniley MS, Livera LN, Wickramasinghe YABD, Rolfe $P$. Non-invasive monitoring of cerebral tissue oxygenation in-vivo by near infra red spectroscopy: a sensitive indicator of oxygenation changes. Biochem Soc Trans 1989;17:903-4.

10 Whitney RJ. The measurement of volume changes in human limbs. F Physiol 1953;121:1-27.

11 Hallbook T, Mansson B, Nilsen R. A strain gauge plethysmograph with electrical calibration. Scand $\mathcal{F}$ Clin Lab Invest 1970;25:413-8.

12 Cooke RWI, Lucas A, Pryse-Davies J, Yudkin PLN. Head circumference as an index of brain weight in the foetus and newborn infant. Early Hum Dev 1977;1:145-9.

13 Cooke RWI, Rolfe P, Howat P. A technique for the noninvasive estimation of cerebral blood flow in the newborn infant. F Med Eng Technol 1977;1:263-6.

14 Conover WJ. Practical non parametric statistics. New York: J Wiley, 1980: 199-308.

15 Levene MI. Cerebral blood flow. In: Neonatal neurology. (Current reviews in paediatrics.) Edinburgh: Churchill Livingstone, 1987:28-35.

16 Greisen G. Methods for assessing cerebral blood flow. In: Levene MI, Punt J, eds. Fetal and neonatal neurology and neurosurgery. Edinburgh: Churchill Livingstone, 1988: 